\title{
Sport als Antidementivum
}

\section{In einer retrospektiven Studie an fast 1000 Frauen über 65 Jahren zeigt sich, dass die körperliche Aktivität im Lauf des Lebens, vor allem aber in der Jugend, ein protektiver Faktor gegen den kognitiven Abbau im Alter darstellt.}

— Obwohl es bereits viele epidemiologische Untersuchungen gibt, die einen präventiven Effekt körperlicher Aktivität gegen Demenz und leichtere kognitive Einschränkungen im Alter gezeigt haben, existieren nur wenige Untersuchungen, die auch die körperliche Aktivität vor der Lebensmitte, und überhaupt keine Studien, die diesen Aspekt zu verschiedenen Zeitpunkten im Leben berücksichtigt haben.

9344 Frauen über 65 Jahren, die in eine Studie zur Untersuchung osteoporotischer Frakturen einbezogen waren, wurden auch über ihre körperliche Aktivität bzw. ihre Inaktivität im Teenageralter sowie im Alter von 30, 50 und im gegenwärtigen Alter befragt. Zu den vier Zeitpunkten bezeichneten sich $15,5,29,7,28,1$ und $21,1 \%$ der Probanden als vollständig körperlich inaktiv.

Die Intensität der körperlichen Aktivität wurde in drei Kategorien eingeteilt. Die unterste Kategorie bestand in regelmäßigem Spazierengehen und Gärtnern, die mittlere in Aktivitäten wie Tanzen oder Tennis und die hohe in Jogging oder Skifahren. Die Assoziation zwischen körperlicher Aktivität und dem Ergebnis einer modifizierten Mini-Mental-State-Examination wurde für die einzelnen Lebensalter statistisch untersucht. Dabei zeigte sich, dass die Wahrscheinlichkeit für eine kognitive Einschränkung im höheren Lebensalter bei den körperlich aktiven Frauen für jedes Lebensalter negativ assoziiert war.

Am ausgeprägtesten war dieser Effekt bei der körperlichen Aktivität im Teenageralter feststellbar. Die Wahrscheinlichkeit für eine kognitive Beeinträchtigung betrug beim Vergleich zwischen den völlig inaktiven und den ak-

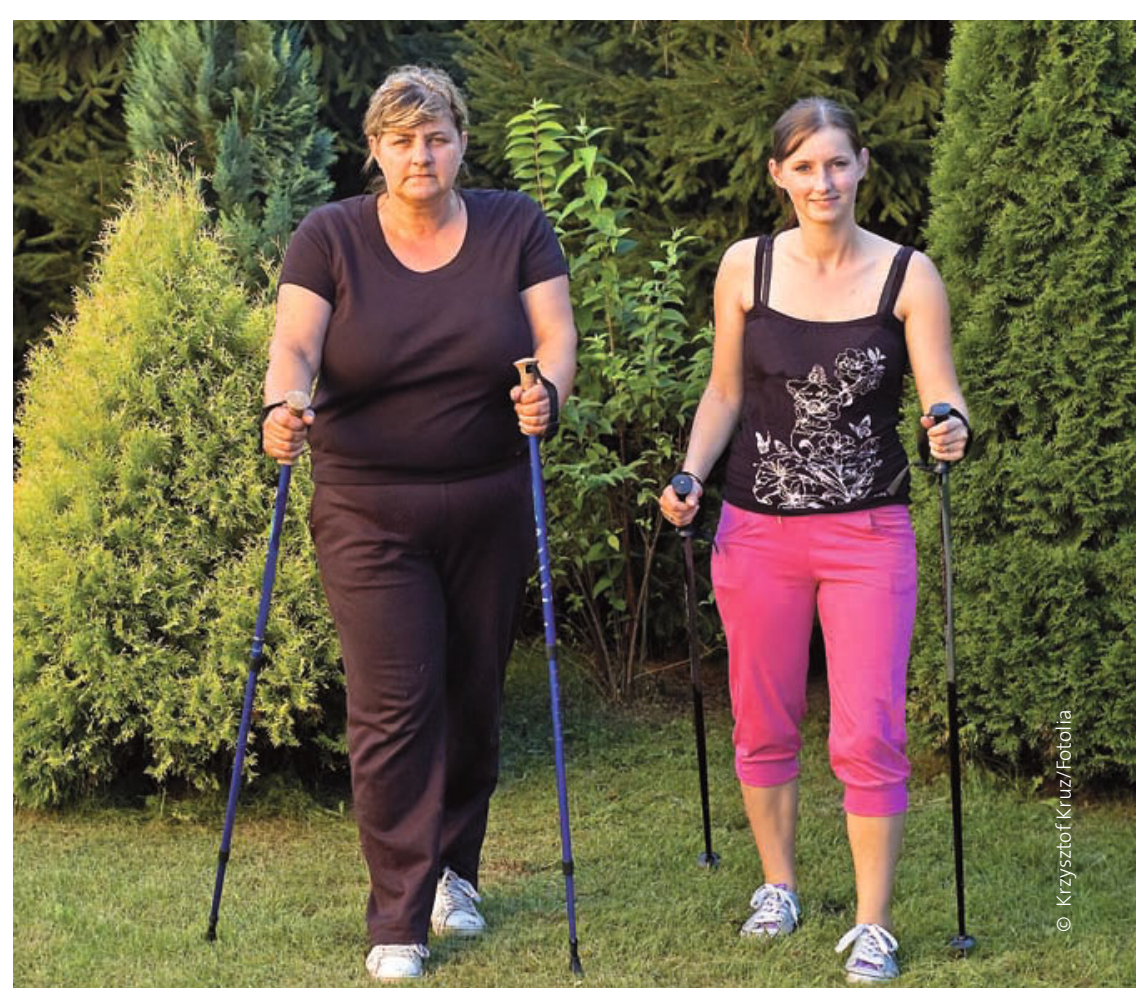

tiven Frauen für das Teenageralter 8,5 vs. $16,7 \%$, für das Alter von 308,9 vs. $12,0 \%$, für das Alter von 508,5 vs. $13,1 \%$ und für das höhere Lebensalter 8,2 vs. $15,9 \%$. Die Assoziation verblieb für jedes Lebensalter signifikant, wenn die Daten für potenzielle konfundierende Faktoren korrigiert wurden.

Wer nun glaubt, es sei alles zu spät, da sein Teenageralter lange vorbei sei, der irrt. Auch Frauen, die als Teenager Couch-Potatoes waren und erst im späteren Leben sportliche Aktivitäten aufgenommen haben, konnten ihr Risiko für kognitive Beeinträchtigung im Alter über 65 ebenfalls noch deutlich reduzieren.

\section{Kommentar}

Die Studien über den positiven Einfluss von körperlicher Aktivität auf nahezu sämtliche Bereiche der Gesundheit füllen mittlerweile ganze Bibliotheken. Niemand kann sagen, wir hätten die Erkenntnis nicht. Unter diesen Umständen wundert es umso mehr, warum wir in den Schulen von der täglichen Sportstunde weiter entfernt sind denn je und unsere Kinder durch die "fortschrittliche“ Lebensweise immer mehr zur Inaktivität verführt, oder sollte man besser sagen, verdammt werden.

H. S. FÜEßL =
- L. E. Middleton et al.

(Korr. : Laura Middleton, 2075 Bayview Ave A408, Toronto, ON, Canada $\mathrm{M}_{4} \mathrm{~N}_{3} \mathrm{M}_{5}$, e-mail : Laura.mittleton@sunnybrook.ca): Physical activity over the life course and its association with cognitive performance and impairment in old age. J. of the Am. Getiatr. Society 58 (2010) 7, 1322-1326

\footnotetext{
4 Auch wenn es für Sport nie zu spät ist: Früher ist besser.
} 